\title{
Desempenho reprodutivo, características do pelame e taxa de sudação em vacas da raça Braford
}

\author{
Elaine Cristina Abaker Bertipaglia ${ }^{1}$, Roberto Gomes da Silva², Vânia Cardoso ${ }^{3}$, Luiz Alberto Fries ${ }^{3 *}$ \\ ${ }^{1}$ Departamento de Reprodução Animal, FCAV-UNESP - CEP: 14884-900 - Jaboticabal. \\ 2 Departamento de Zootecnia, FCAV-UNESP - Jaboticabal. \\ ${ }^{3}$ Gensys consultores associados S/S LTDA. \\ *In memoriam.
}

RESUMO - Foram investigados os efeitos das características do pelame e da taxa de sudação (TS) sobre o intervalo de partos (IEP, $n=423$ ) e os dias para o parto (DPP, $n=1.202$ ) de vacas da raça Braford criadas em sistema extensivo em região tropical úmida no Mato Grosso do Sul, Brasil. As análises foram realizadas pelo método dos quadrados mínimos considerando para intervalo de partos os seguintes efeitos das classes de idade da vaca ao parto: grupo genético; pai; e regressão sobre a refletância da superfície da capa (R), a espessura da capa (E), o comprimento dos pêlos (C), o número de pêlos por unidade de área (N), o diâmetro dos pêlos (D) e a taxa de sudação. Para dias para o parto, foram considerados os efeitos: R, E, C, N, D, TS, divididos em classes; grupo genético; idade no início da estação de monta; e pai. Os componentes de variância e co-variância foram estimados pelo método de Máxima Verossimilhança Restrita sob o modelo touro. As características adaptativas não foram importantes para a variação do intervalo de parto e dos dias para o parto, mas a taxa de sudação apresentou efeito importante sobre dias ao parto. As estimativas de herdabilidade foram quase nulas para IEP e DPP e maiores para as características adaptativas $(\mathrm{E}=0,16 \pm 0,09 ; \mathrm{C}=0,18 \pm 0,09 ; \mathrm{N}=0,08 \pm 0,06 ; \mathrm{D}=0,12 \pm 0,07 ; \mathrm{R}=0,30 \pm 0,12 ; \mathrm{TS}=0,10 \pm 0,06$ ). Os resultados obtidos para as correlações genéticas de IEP e DPP com TS e entre as características do pelame indicaram que a seleção simultânea para menores valores de C pode ser favorável à redução do intervalo de partos. Em geral, as correlações genéticas entre TS, IEP e DPP apresentaram elevado erro-padrão. Considerando os resultados deste estudo e a importância das características do pelame e da sudação, confirma-se a necessidade de se estudarem as correlações genéticas entre as características adaptativas e o desempenho reprodutivo para o progresso da adaptação de bovinos da raça Braford no Brasil.

Palavras-chave: Braford, gado de corte, herdabilidade, pelame, reprodução, sudação

\section{Effects of hair coat traits and sweating rate on reproductive performance of Braford cows}

\begin{abstract}
This study aimed to evaluate the effects of hair coat traits (thickness (T), reflectance (R), length (L), diameter (D) and number of hairs per unit area $(\mathrm{N})$ ) and sweating rate $(\mathrm{SR})$ on calving interval $(\mathrm{CI}, \mathrm{n}=423)$ and days to calving (DC, $n=1202$ ) of Braford cows raised in Mato Grosso do Sul State, Brazil. Data were analyzed by least squares and the model for CI included the effects of age class of cow, genetic group, sire and regression on R, T, L, N, D and SR. For days to calving the following effects were considered: R, T, L, N, D, TS divided in classes; regressions on genetic group, age at the beginning of breeding season and sire. Variance and covariance components were estimated by REML. Hair coat traits and SR traits were not statistically significant for CI, but the SR effect was statistically significant on DC. The heritability estimates were practically null for CI and DC and ranged from 0.08 to 0.30 for adaptive characteristics $(\mathrm{T}=0.16 \pm 0.09$; $\mathrm{L}=0.18 \pm 0.09$; $\mathrm{N}=0.08 \pm 0.06 ; \mathrm{D}=0.12 \pm 0.07 ; \mathrm{R}=0.30 \pm 0.12 ; \mathrm{SR}=0.10 \pm 0.06$ ). Overall, estimates of genetic correlations between pair of traits (hair coat, sweating rate, CI and DC) were characterized by high standard errors. Additional data is required to obtain accurate estimates of genetic correlations among hair coat traits, sweating rate and reproductive performance of Braford cows in Brazil.
\end{abstract}

Key Words: beef cattle, Braford, hair coat, heritability, reproduction, sweating rate

\section{Introdução}

A eficiência reprodutiva de bovinos em ambientes tropicais é baixa se comparada ao desempenho em ambientes temperados. O melhoramento desta característica em rebanhos sob condições extensivas em áreas tropicais é difícil, em razão dos efeitos do estresse térmico e da subnutrição periódica sobre o desempenho de animais de

Este artigo foi recebido em 12/3/2007 e aprovado em 13/3/2008.

Correspondências devem ser enviadas para ecabertipaglia@yahoo.com.br. 
alto potencial de produção (Finch, 1984). As interações dos mecanismos reprodutivos com fatores genéticos e ambientais têm recebido a atenção de diversos pesquisadores (Ortovant et al., 1988; Becerril et al., 1993; Burrow, 2001), mas há muito pouca informação sobre a influência de fatores associados à adaptação ao ambiente tropical. Programas de seleção envolvendo variáveis associadas à adaptação têm sido sugeridos para o melhoramento produtivo de bovinos em áreas tropicais (Silva, 1973; Prayaga, 2003; Bueno et al., 2006).

O estresse térmico é um dos fatores associados à falha na gestação e seus efeitos sobre a reprodução de vacas comprometem a esteroidogênese, pois podem ocasionar alterações na duração e no comportamento sexual durante o estro (Nebel et al., 1997) e aumento da ocorrência de oócitos anormais (Hansen, 2002) e de morte embrionária precoce (Putney et al., 1989; Sartori et al., 2002) e, conseqüentemente, redução significativa nas taxas de concepção (Badinga et al., 1985; Hansen, 1997).

Da fase embrionária de zigoto até antes do desenvolvimento da mórula do embrião, é significativa a alteração do ambiente uterino, especialmente, a hipertermia (Edwards et al., 2001). Com o avançar da gestação, a partir do terceiro dia (8 a 16 células), ocorre maior síntese protéica (Barnes \& Eyestone, 1990), sobretudo das proteínas antichoque térmico.

Características da superfície corporal externa (espessura da capa de pelame, número de pêlos por unidade de área, diâmetro e comprimento dos pêlos e cor do pelame) afetam as trocas de calor nos animais (Cena \& Monteith, 1975; McArthur, 1991; Silva, 1999). Além dessas características, as propriedades radiativas do pelame (refletância, transmitância, absortância) são muito importantes para animais em ambientes abertos, como em pastagem, e condicionam a resistência à radiação solar (Silva, 2000a). Em geral, segundo Silva (1999), o tipo mais adequado de bovino para regiões tropicais seria aquele com capa de pelame branco, pouco espessa, com pêlos curtos, grossos, numerosos e bem assentados sobre uma epiderme altamente pigmentada.

O resfriamento por evaporação cutânea, um dos mais importantes meios de controle térmico nos bovinos, é promovido pela sudação e favorecido pela movimentação do ar e morfologia do pelame (Silva, 1999; Gebremedhin $\& \mathrm{Wu}, 2002)$.

Apesar do interesse econômico da raça Braford no Brasil, sob o ponto de vista da qualidade de carcaça, da precocidade sexual e do ganho de peso, pouco estudos têm sido realizados para analisar os efeitos das características adaptativas sobre o desempenho reprodutivo. Desse modo, realizou-se este estudo para investigar os efeitos das características do pelame e da taxa de sudação sobre o desempenho reprodutivo de vacas da raça Braford, juntamente com a estimação dos parâmetros genéticos dessas características, visando fornecer subsídios para programas de melhoramento genético.

\section{Material e Métodos}

Os dados de intervalo de partos e dias para o parto de 423 e 1.202 animais, respectivamente, foram obtidos no rebanho de vacas Braford da Fazenda Dom Arlindo, em

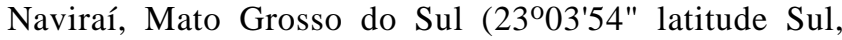
54¹1'26" longitude Oeste, $403 \mathrm{~m}$ de altitude). Esta região apresenta clima tropical úmido, caracterizado por invernos secos e verões chuvosos e quentes. A média mensal da temperatura máxima do ar variou de 21 a $33^{\circ} \mathrm{C}$. A estação chuvosa inclui os meses de setembro a abril e a estação seca ocorre no inverno (maio a agosto). A umidade relativa do ar varia de 60 a 75\%. Durante o verão, as altas temperaturas, combinadas às elevadas taxas de umidade e à intensa radiação solar, geram condições bastante estressantes.

Ao nascimento, os animais foram identificados e submetidos a procedimentos sanitários preventivos, considerando as características epidemiológicas da região, com destaque para o controle de endo e ectoparasitos. Os animais foram manejados exclusivamente em pastagem (Brachiaria brizantha), sem áreas de sombras e com suplementação mineral. A propriedade é associada à Conexão Delta G, um programa de melhoramento genético das raças Braford, Hereford e Nelore, cujo objetivo é a seleção de machos para precocidade sexual e de ganho de peso. Todas as novilhas não-gestantes ao final da estação de monta de outubro a dezembro são inseminadas na estação seguinte (abril a maio). O rebanho é composto de animais com diferentes combinações Hereford e Nelore $(\mathrm{Ne})$ e varia de acordo com sua porcentagem de Hereford, de 25 a $75 \%$. Algumas vacas nascidas em 1998 e 1999 com composições raciais 25 e $50 \%$ Hereford nasceram na Fazenda Cedro (Fazendas Paquetá Ltda, localizada em Ponta Porã, Mato Grosso do Sul), de onde foram trazidas quando novilhas para a fazenda Don Arlindo, cujo rebanho foi estabelecido recentemente.

Os animais em estudo incluíam novilhas, vacas com bezerros (em lactação) e sem bezerros (não-lactantes), com 1 a 5 anos de idade, respectivamente, todas filhas de 25 touros, inclusive reprodutores múltiplos (24 com identificação). Os animais eram manejados em 16 grupos de acordo 
com a composição racial, o ano de nascimento e a categoria (novilhas, vacas com bezerra, vacas com bezerro, vacas sem bezerros) em três diferentes retiros da Fazenda.

Ao atingir $260 \mathrm{~kg}$ (306 a 527 dias de idade), as novilhas eram consideradas aptas à reprodução. A estação de monta era de 60 dias: 30 dias com inseminação artificial e o resto com diversos touros. Para as vacas com ou sem bezerros, a estação de monta foi de 90 dias ou menos, conforme o número de animais inseminados no lote e a porcentagem de manifestação de cio. A detecção de cio era realizada por observação visual no início da manhã e ao final da tarde, utilizando-se rufiões. Doze horas depois, era feita a inseminação e aos 60 dias após o término da estação de monta era feito diagnóstico de gestação por palpação retal. Vacas não-gestantes são descartadas do rebanho. Uma segunda oportunidade é dada somente às novilhas, as quais são expostas à reprodução na estação de monta seguinte (abril a maio), de modo que há duas estações de monta anuais. A variável dias para o parto foi considerada conforme sugerido por outros autores (Bourdon \& Brinks,1983; MacGregor, 1995), considerando o uso de estação de monta restrita. O intervalo de partos foi considerado por ser uma variável amplamente utilizada, principalmente em rebanhos com apenas dados dos partos.

O intervalo de partos foi calculado como o número de dias entre os partos dos anos de 2003 e 2004, ocorridos entre final de julho ao início de novembro, no caso de animais com dois ou mais partos consecutivos. A característica dias para o parto ( DPP) foi definida de acordo com Johnston \& Bunter (1996), como o número de dias entre a primeira observação de cio e o parto de vacas e novilhas, incluindo somente o período da estação de monta e os nascimentos nos anos de 2003 e 2004. Foram atribuídas penalidades às vacas que falharam em parir: o valor máximo de DPP foi somado de 21 dias (um período do ciclo estral), conforme descrito por Johnston \& Bunter (1996), para cada grupo de manejo na estação de monta. A correção para o sexo do bezerro foi feita somando-se um dia ao DPP para fêmeas, de acordo com o tempo de gestação para cada sexo em mestiços NeloreHereford (Rocha et al., 2005). Para fêmeas que não pariram, não foi realizada a correção para o sexo. Foram considerados valores de DPP superiores a 277, que corresponde ao período gestacional médio $(289,66 \pm 13,3)$ menos o erro-padrão.

Analisaram-se a espessura (E, mm) e refletância da capa $(\mathrm{R}, \%)$, o comprimento médio $(\mathrm{C}, \mathrm{mm})$, o número por unidade de área $\left(\mathrm{N}\right.$, pêlos $\left./ \mathrm{cm}^{2}\right)$ e o diâmetro médio $(\mathrm{D}, \mathrm{mm})$ dos pêlos. As medições do pelame e a coleta de amostras de pêlos dos animais foram realizadas no período de 27 de outubro a 21 de dezembro de 2003. As amostras de pêlos foram retiradas
$20 \mathrm{~cm}$ abaixo da coluna vertebral, na região torácica mediana, utilizando-se o método descrito por Silva (2000a).

As características do pelame foram determinadas conforme métodos descritos por Silva (2000a). A espessura da capa de pelame foi avaliada diretamente no local de amostragem dos pêlos utilizando-se uma régua metálica milimetrada provida de um cursor. O comprimento dos pêlos foi determinado como a média aritmética do comprimento dos dez maiores pêlos da amostra, selecionados por análise visual e medidos com paquímetro digital. O número de pêlos por $\mathrm{cm}^{2}$ foi estimado pela contagem do número de fibras da amostra, correspondente a $18 \mathrm{~mm}^{2}$, da superfície da pele do animal. Em seguida, realizou-se a conversão para número de pêlos por $\mathrm{cm}^{2}$. O diâmetro médio foi determinado com micrômetro digital, considerando a média aritmética dos diâmetros dos dez maiores pêlos da amostra.

A refletância da capa do pelame $(R)$ foi definida de acordo com a seguinte fórmula:

$$
\begin{gathered}
\mathrm{R}=\mathrm{r}_{\text {vermelho }}(\mathrm{Pg})(\mathrm{Cp})+\mathrm{r}_{\text {preto }}(\mathrm{Cp})+\mathrm{r}_{\text {branco }}(\mathrm{Cp})+ \\
\mathrm{r}_{\text {cinzento }}(\mathrm{Cp})(\mathrm{Pg})+\mathrm{r}_{\text {cinza }}(\mathrm{Cp})
\end{gathered}
$$

em que cada valor $r$ refere-se à refletância $(\%)$ do pelame para cada cor (vermelho $=0,513$; preto $=0,121$; branco $=$ 0,$621 ;$ cinza $=0,512$; cinzento $=0,340$ ) determinada para comprimentos de onda de 350 a $850 \mathrm{~nm}$, conforme resultados descritos por Silva et al. (2003). A combinação de cores do pelame $(\mathrm{Cp})$ foi definida como a proporção de manchas vermelhas, pretas, brancas, cinzas ou cinzentas sobre a superfície total do animal. Cinzenta era a cor do pelame constituída de pelos brancos misturados com pretos. A pigmentação do pelame ( $\mathrm{Pg}, \%)$ era o tom ou a intensidade da cor (vermelho ou cinzento, em porcentagem) determinada por avaliação visual direta do animal, comparando-se com uma escala padrão colorida impressa (Silva, 2000a). O padrão era colocado sobre a superfície a ser avaliada, atribuindo-se um valor de 0 a 100, conforme a coincidência das tonalidades (do branco puro aos tons de cinza a preto; ou do baio ao vermelho intenso) do pelame e do padrão. Não foram considerados os membros cauda, cabeça e região inferior abdominal e torácica.

A taxa de sudação foi determinada pelo método de Schleger \& Turner (1965). As medidas foram realizadas diariamente, das $8 \mathrm{~h}$ às $18 \mathrm{~h}$, no mês de fevereiro de 2004 . Eram registrados os dados de mesmo grupo de manejo em mesmo dia, evitando-se dias de chuva. Os animais eram trazidos para o curral por volta das 7-8 horas ou 13-14 horas, onde eram mantidos sem acesso a sombra. As medidas eram feitas 30 minutos depois de os animais chegarem no curral para permitir que descansassem. A contenção dos animais era feita somente no momento da observação. 
Para cada observação da taxa de sudação de todas fêmeas (IEP = 423 e DPP = 1202), foram obtidas medidas da temperatura do ar, da pressão parcial de vapor e da carga térmica radiante, calculadas como descrito por Silva (2000a). A taxa de sudação foi ajustada por regressão sobre as mesmas condições de temperatura do ar $\left(31^{\circ} \mathrm{C}\right)$, pressão parcial de vapor $(2,00 \mathrm{kPa})$, carga térmica radiante $\left(651 \mathrm{Wm}^{-2}\right)$ e hora da observação $(13 \mathrm{~h})$, considerando a variável tanto dependente como independente, nos modelos de IEP e DPP.

Os dados foram inicialmente analisados pelo método dos quadrados mínimos (Littell et al., 1991), após confirmação de distribuição normal pelo teste de Shapiro-Wilk. A multicolinearidade entre as variáveis independentes nos modelos foi investigada em modelo de regressão pelos fatores de inflação de variância (FIV). Foram retiradas as variáveis que apresentaram alto valor da variância de inflação para o modelo de regressão (PROC REG; vif, do programa SAS).

O modelo para o intervalo de partos incluiu os seguintes efeitos fixos: efeitos lineares e quadráticos da refletância do pelame; efeitos lineares da espessura do pelame, do comprimento dos pêlos, do número de pêlos por unidade de área, do diâmetro dos pêlos e da taxa de sudação; efeito de classes da idade da vaca ao parto no ano de 2003 (6 classes, $1=1.017-1.091 \mathrm{dias} ; 2=1.092-1.166 \mathrm{dias} ; 3=1.401-1.484 \mathrm{dias}$ $4=1.485-1.567$ dias $; 5=1.750-1.832$ dias $; 6=1.833-1.915$ dias); efeito de classe dos grupos genéticos ( 3 classes, $1=$ $25 \% \mathrm{He} ; 2=50 \% \mathrm{He} ; 3=62,5 \% \mathrm{He}$ ); efeito de classes do pai da vaca ( 25 touros).

A variável dias para o parto incluiu os seguintes efeitos fixos: classes da refletância (três classes, $1=0,063-0,194 \%$; $2=0,195-0,446 \% ; 3=0,446-0,626 \%)$; classes do número de pêlos (três classes, $1=450-1.660$ pêlos $/ \mathrm{cm}^{2} ; 2=1.661-3.010$ pêlos $/ \mathrm{cm}^{2} ; 3=3.011-4.851$ pêlos $/ \mathrm{cm}^{2}$ ); classes do diâmetro dos pêlos (três classes, $1=10-31 \mu \mathrm{m} ; 2=32-41 \mu \mathrm{m} ; 3=$ 42-71 $\mu \mathrm{m}$ ); classes do comprimento dos pêlos (três classes, $1=280-800 \mathrm{~mm} ; 2=801-1.533 \mathrm{~mm} ; 3=1.534-2.093 \mathrm{~mm}$ ); classes de espessura do pelame (três classes; 1 = 1-3 mm; $2=4-6 \mathrm{~mm} ; 3=7-9 \mathrm{~mm}$ ); classes da taxa de sudação (três classes, $1=135-320 \mathrm{~g} \cdot \mathrm{m}^{-2} \mathrm{~h}^{-1} ; 2=321-590 \mathrm{~g} \cdot \mathrm{m}^{-2} \mathrm{~h}^{-1} ; 3=592$ 860 g. $\mathrm{m}^{-2} \mathrm{~h}^{-1}$ ); classes de grupo genético (quatro classes; $1=25 \% \mathrm{He} ; 2=37,5 \%$ Hereford $3=50 \%$ Hereford $4=62,5 \%$ Hereford); efeito de classes da idade no início da estação de monta ( seis classes, $1=345-525$ dias, $2=526-705$ dias, 3 =706-885 dias, $4=886-1.245$ dias, $5=1.246-1.605$ dias e $6=$ 1.606-1.965 dias); efeito de classe do pai (31 touros).

As estimativas dos parâmetros genéticos, dos componentes de variância e co-variância, das herdabilidades e das correlações genéticas foram realizadas pelo método da Máxima Verossimilhança Restrita (REML), sob o modelo touro, utilizando-se o programa Multiple Trait Derivative Free Restricted Maximum Likelihood (MTDFREML). Vacas filhas de pais desconhecidos ou reprodutores múltiplos foram eliminadas destas análises.

Os modelos para estimação dos parâmetros genéticos foram os mesmos utilizados para obtenção dos valores iniciais dos componentes da variância, considerando apenas os efeitos que foram significativos $(\mathrm{P}<0,05)$ para o IEP e DPP. Para as características do pelame e taxa de sudação, foram considerados os mesmos modelos analisados e efeitos fixos significativos (idade do animal, mês da coleta, grupo genético, grupo de contemporâneos, pai) descritos por Bertipaglia et al. (2007b) para os mesmos animais e a mesma base de dados deste estudo. Os valores iniciais de cada componente de variância pedido pelo programa MTDFREML (Boldman et al., 2002) para as análises univariadas foram obtidos de estudos anteriores.

A equação de modelo misto de touro para as características do pelame, taxa de sudação, dias para o parto e intervalo de partos para análises univariadas em notação matricial foi:

$$
\mathrm{y}=\mathrm{I} \mu+\mathrm{X} \beta+\mathrm{Zs}+\mathrm{e}
$$

em que: $y=n x 1$ vetor de observações para cada uma das variáveis (espessura do pelame, refletância, comprimento, diâmetro e número de pêlos, taxa de sudação, dias para o parto e intervalo de partos); $\mu=$ média geral; $I=$ vetor $n \times 1$ para todas entradas para cada característica; $X=$ matriz de incidência nxp associada aos p efeitos fixos; $\beta=$ vetor $\mathrm{px} 1$ de efeitos fixos; $Z=$ matriz de incidência $n x t$ associada aos $\mathrm{t}$ efeitos aleatórios de touro; $\mathrm{s}=$ vetor $\mathrm{tx} 1$ do efeito aleatório genético do touro; e e = vetor $\mathrm{nx} 1$ de efeitos aleatórios do resíduo. Outras definições: $\mathrm{n}=$ número total de vacas com medidas (IEP, $\mathrm{n}=274$; DPP, $\mathrm{n}=616$ ); $\mathrm{t}=$ número de touros (IEP, $\mathrm{t}=24$; DPP, $\mathrm{t}=30$ ); e $\mathrm{p}=$ número de níveis dos efeitos fixos.

As pressuposições dos modelos foram: $\mathrm{E}(\mathrm{y})=\mathrm{X} \mu$; $\mathrm{E}(\mathrm{s})=0 ; \mathrm{E}(\mathrm{e})=0 ; \operatorname{Var}(\mathrm{s})=\mathrm{I}_{\mathrm{n}} \sigma^{2}{ }_{\mathrm{s}}=1 / 4 \mathrm{I}_{\mathrm{n}} \sigma^{2}{ }_{\mathrm{a}} ; \operatorname{Var}(\mathrm{e})=\mathrm{I}_{\mathrm{n}} \sigma^{2}{ }_{\mathrm{e}}$, em que $I_{n}=$ matriz identidade; $\sigma^{2}{ }_{s}=$ variância genética aditiva do touro; $\sigma_{\mathrm{a}}^{2}=$ variância genética aditiva; e $\sigma_{\mathrm{e}}^{2}=$ variância residual.

Um modelo misto sem o efeito fixo do genótipo e somente o efeito aleatório de touro foi usado para estimar a herdabilidade no sentido amplo $\left(\mathrm{H}^{2}\right)$. A herdabilidade no sentido estrito $\left(\mathrm{h}^{2}\right)$ foi estimada pelo modelo touro contendo todos os efeitos fixos em adição do genótipo (contribuição dos genes do Hereford para a composição genética de cada animal). Para as análises bivariadas, o modelo touro foi: 


$$
\mathrm{y}=\mathrm{X} \beta+\mathrm{Zs}+\mathrm{E}
$$

em que $\mathrm{Y}=\left[\mathrm{y}_{1} \mathrm{y}_{2}\right]=$ matriz $\mathrm{nx} 2$ de observações das variáveis 1 e 2 em análise; $B=\left[\beta_{1} \beta_{2}\right]=$ matriz px 2 de efeitos fixos das variáveis 1 e $2 ; \mathrm{S}=\left[\mathrm{s}_{1} \mathrm{~s}_{2}\right]=$ matriz tx 2 de efeitos aleatórios aditivos do touro das variáveis 1 e $2 ; E=\left[\mathrm{e}_{1} \mathrm{e}_{2}\right]=$ matriz $\mathrm{nx} 2$ dos efeitos aleatórios residuais das variáveis 1 e 2 . As pressuposições são:

$\mathrm{E}[\mathrm{y}]=\mathrm{XB}$;

$\operatorname{Var}(\mathrm{S})=\mathrm{I}_{\mathrm{n}} \otimes\left[\begin{array}{ll}\sigma_{\mathrm{s}_{11}} & \sigma_{s_{12}} \\ \sigma_{s_{21}} & \sigma_{s_{22}}\end{array}\right]=\mathrm{I}_{\mathrm{n}} \otimes(1 / 4)\left[\begin{array}{l}\sigma_{\mathrm{a}_{11}} \sigma_{a_{12}} \\ \sigma_{\mathrm{a}_{21}} \sigma_{a_{22}}\end{array}\right] \mathrm{e}$

$\operatorname{Var}(\mathrm{E})=\mathrm{I}_{\mathrm{n}} \otimes\left[\begin{array}{ll}\sigma_{1}^{2} & 0 \\ 0 & \sigma_{2}^{2}\end{array}\right]$,

em que $\otimes=$ produto direto entre duas matrizes (produto de Kronecker); $\mathrm{I}_{\mathrm{n}}=$ matrizes de identidade. $\mathrm{O}$ processo iterativo era considerado convergente quando a variância dos valores da função $(-2 \log L)$ do simplex era menor que o critério de convergência $\left(1 \times 10^{-9}\right)$.

\section{Resultados e Discussão}

O intervalo de partos médio $(381,61 \pm 26,34$ dias, Tabela 1) foi inferior ao de 404,6 $\pm 5,44$ dias observado por Oliveira et al. (2002) em vacas Brangus Ibagé (3/8 Nelore 5/8 Angus), classificadas como de alta fertilidade, e $523,5 \pm 4,77$ dias para aquelas de mediana fertilidade, ambos os grupos manejados em estação de monta de 90 dias. Também foi menor que os 437,3 $\pm 6,3$ dias para vacas Hereford no Rio Grande do Sul (Moreira \& Cardelino, 1994) e 446 dias para Nelore em Mato Grosso do Sul (Silveira et al., 2004).

Valores de intervalo de parto menores indicam fêmeas mais férteis e eficientes. Entretanto, em gado de corte, é comum o uso de estação de monta definida, o que leva a uma interpretação equivocada dos resultados, motivo pelo qual MacGregor (1995) sugeriu associação negativa com a data do parto anterior. Bourdon \& Brinks (1983) consideraram desvantajoso o uso do intervalo de partos como critério de seleção, uma vez que selecionaria intervalos mais curtos e levaria à seleção indireta de animais tardios, pois vacas que parem no final da estação de monta podem parir em intervalo mais curto em comparação a suas contemporâneas que pariram no início da estação.

O valor médio de DPP neste estudo foi de 336,69 $\pm 41,16$ dias, incluindo fêmeas que não pariram (Tabela 1). Desse valor, 289,66 \pm 13,3 dias corresponderam à duração da gestação média e os demais 47 dias ao período de serviço.

A taxa média de sudação foi $493,59 \mathrm{~g} \mathrm{~m}^{-2} \mathrm{~h}^{-1}$ para os 423 animais sob análise do intervalo de partos e $484,32 \mathrm{~g} \mathrm{~m}^{-2} \mathrm{~h}^{-1}$ para os 1202 analisados para DPP. Esses valores são altos e ótimos para regiões tropicais e próximos dos observados por Schleger \& Turner (1965) para novilhos Hereford $\times$ Shorthorn $\left(488 \mathrm{~g} \cdot \mathrm{m}^{-2} \mathrm{~h}^{-1}\right)$ e Brahman $\times$ Shorthorn $\left(500\right.$ g. $\left.\mathrm{m}^{-2} \mathrm{~h}^{-1}\right)$ no verão australiano. Esses resultados foram superiores aos observados por Silva et al (1988) em gado Jersey e por Finch et al. (1982) em mestiços Hereford $\times$ Shorthorn $\times$ Brahman na Austrália. Estudo recente realizado no Brasil Central por McManus et al. (2005) evidenciou taxas de sudação mais baixas para vacas da raça Nelore $\left(215,22 \mathrm{gm}^{-2} \mathrm{~h}^{-1}\right)$ e Holandesa $\left(215,22\right.$ e $\left.269,13 \mathrm{gm}^{-2} \mathrm{~h}^{-1}\right)$, que podem ser atribuídas o fato de que os animais da raça Nelore, como todos os zebuínos, tendem a elevar a taxa de sudação apenas sob temperaturas ambientes muito altas.

As 1.202 vacas analisadas para DPP apresentaram pelame com refletância média, semelhante à da cor cinzenta do Nelore (0,34, segundo Silva et al., 2003). Na raça Braford, ocorrem pelames de cor vermelha com grande proporção de pêlos negros (brasina), com maior absortância e menor refletância da radiação solar, resultando em maior aquecimento da superfície corporal. Entretanto, esse maior

Tabela 1 - Intervalo de partos (IEP), dias para o parto (DPP), taxa de sudação e características do pelame de vacas Braford

\begin{tabular}{|c|c|c|c|c|c|c|}
\hline \multirow[t]{2}{*}{ Variável } & \multicolumn{3}{|c|}{ IEP $(n=423)$} & \multicolumn{3}{|c|}{ DPP $(\mathrm{n}=1.202)$} \\
\hline & Média & Min & $\operatorname{Max}$ & Média & Min & Max \\
\hline IEP & $381,61 \pm 26,34$ & 295,00 & 453,00 & - & - & - \\
\hline DPP & - & - & - & $336,69 \pm 416$ & 277 & 440 \\
\hline TS & $493,59 \pm 120,52$ & 175,33 & 845,62 & $484,32 \pm 126,75$ & 135,68 & 938,01 \\
\hline E & $3,14 \pm 1,25$ & 1,00 & 8,00 & $3,60 \pm 1,65$ & 1,00 & 13,00 \\
\hline $\mathrm{N}$ & $916 \pm 409$ & 324 & 3456 & $965 \pm 496$ & 319 & 4851 \\
\hline $\mathrm{C}$ & $866,34 \pm 222,60$ & 413,40 & 1780,40 & $1.004,50 \pm 336,97$ & 413,40 & $2.650,40$ \\
\hline D & $28,74 \pm 7,21$ & 12,00 & 60,00 & $31,17 \pm 8,31$ & 11 & 69 \\
\hline $\mathrm{R}$ & $0,17 \pm 0,13$ & 0,0051 & 0,6300 & $0,3214 \pm 0,8385$ & 0,0892 & 0,5915 \\
\hline
\end{tabular}

IEP = intervalo de partos (dias); DPP = dias para o parto; TS = taxa de sudação $\left(\mathrm{gm}-{ }^{2} \mathrm{~h}-{ }^{1}\right) ; \mathrm{E}=$ espessura da capa de pelame $(\mathrm{mm}) ; \mathrm{C}=\mathrm{comprimento}(\mathrm{mm}) ;$ $\mathrm{N}=$ número de pêlos por unidade de área $\left(\right.$ pêlos $\left./ \mathrm{cm}^{2}\right) ; \mathrm{D}=$ diâmetro $(\mu \mathrm{m}) ; \mathrm{R}=$ refletância $(\%)$. 
aquecimento pode ser compensado pela ocorrência de taxas de sudação mais elevadas e pêlos mais curtos, grossos e bem assentados, o que favorece trocas de calor por evaporação cutânea, convecção e condução do calor corporal para o ambiente. De acordo com Silva (1999) e Silva et al. (2001), a seleção de bovinos para maior tolerância ao calor em ambiente tropical deve ser direcionada para pelames predominantemente pretos, no caso de raças nas quais a pigmentação da epiderme acompanha a do pelame, o que ocorre na raça Holandesa, por exemplo. Em ambiente tropical, é fundamental a proteção contra os altos níveis de radiação ultravioleta, proporcionada pela melanina da epiderme e/ou do pelame.

A espessura média do pelame foi pequena $(3,6 \mathrm{~mm}) \mathrm{e}$ com alta densidade de pêlos ( 965 pêlos $/ \mathrm{cm}^{2}$ ). Esses pêlos apresentaram comprimento moderadamente longo $(10,04 \mathrm{~mm})$, fato compensado pelo assentamento sobre a epiderme. O diâmetro médio 31,17 $\mu \mathrm{m}$ também é fino, característico de raças européias. No conjunto, há indícios de um processo de adequação do pelame do Braford às condições do ambiente tropical, em comparação às características da raça Hereford, que, no Canadá, apresentam pêlos de 29,1 mm de comprimento e diâmetro de $45 \mu \mathrm{m}$ (Peter \& Slen, 1964). Uma evolução similar parece ter ocorrido na raça Holandesa. Vacas manejadas em confinamento no Brasil apresentaram capa fina $(2,64 \mathrm{~mm})$, densa (1050 pêlos/ $\left.\mathrm{cm}^{2}\right)$ e com pêlos relativamente curtos $(12,81 \mathrm{~mm})$ e grossos $(62,98 \mu \mathrm{m})$, conforme descrito por Bertipaglia et al. (2005).

Todos os efeitos avaliados para o intervalo de partos foram significativos (Tabela 2), exceto a taxa de sudação e refletância linear para a soma de quadrados (S.Q. I), a qual ignora demais efeitos. O efeito de maior importância foi

Tabela 2 - Resumo da análise de variância do intervalo de partos de vacas Braford

\begin{tabular}{lcrrr}
\hline Fonte & $\mathrm{gl}$ & \multicolumn{1}{c}{ SQ I } & SQ III & \multicolumn{1}{c}{ QM III } \\
\hline R linear & 1 & $0,08^{\mathrm{NS}}$ & 37,78 & $37,78^{\mathrm{NS}}$ \\
R quadrático & 1 & $9.691,52^{* *}$ & 37,81 & $37,81^{\mathrm{NS}}$ \\
E linear & 1 & $3.563,85^{* *}$ & 42,30 & $42,29^{\mathrm{NS}}$ \\
C linear & 1 & $4.251,88^{* *}$ & 135,148 & $135,14^{\mathrm{NS}}$ \\
$\mathrm{N}$ linear & 1 & $6.219,78^{* *}$ & 627,41 & $627,41^{\mathrm{NS}}$ \\
D linear & 1 & $2.592,33^{*}$ & 57,40 & $57,40^{\mathrm{NS}}$ \\
TS linear & 1 & $85,10^{\mathrm{NS}}$ & 258,75 & $258,75^{\mathrm{NS}}$ \\
Idade & 5 & $11.056,71^{* *}$ & $10.818,58$ & $2.163,71^{* *}$ \\
Genótipo & 2 & $8.971,95^{* *}$ & $8.971,95$ & $4.485,97^{* *}$ \\
Pai & 24 & $27.263,58^{* *}$ & $14.627,60$ & $609,48^{\mathrm{NS}}$ \\
Resíduo & 384 & & $219.147,41$ & 570,69 \\
$\mathrm{R}^{2}(\%)$ & 25,17 & & & \\
CV & 6,26 & & & \\
\hline
\end{tabular}

NS $P>0,05 ;{ }^{*} 0,01 \leq 0,05 ;{ }^{* *} \mathrm{P}<0,01 \mathrm{TS}=$ taxa de sudação; $E=$ espessura do pelame; $\mathrm{C}=$ comprimento dos pêlos; $\mathrm{D}$ = diâmetro dos pêlos; $\mathrm{N}=$ número de pêlos por unidade de área; $R$ = refletância da superficie do pelame. refletância (quadrática). Entretanto, a soma de quadrados em análise tipo III (S.Q. III), que considera a importância do efeito depois de todos os outros efeitos (parcial), para o intervalo de partos não apresentou efeito significativo (Tabela 2) para as características do pelame. Esses resultados indicam que, quando considerados em conjunto, os efeitos das características do pelame foram reduzidos, predominando o efeito da composição genética. É possível que pelo menos parte da variação das características do pelame seja decorrente de diferenças entre as composições raciais (genótipo). Na ausência de outros estudos semelhantes a este, esses resultados podem servir de orientação para futuros estudos com rebanhos de raça pura.

$\mathrm{O}$ intervalo de partos dos animais com maior proporção de Nelore $(0,25 \%$ Hereford 0,75 Nelore) foi menor (362,34 dias) que o observado nos demais genótipos. Vacas 0,25 Hereford apresentaram menor intervalo de partos $(362,34$ dias; $\mathrm{P}<0,05)$ em comparação às 50 e 62,5\% Hereford (388,20 e 397,84, respectivamente). Não foi observada diferença significativa entre os animais 50 e $62,5 \%$ Hereford. O melhor desempenho reprodutivo de animais com maior proporção de Nelore indica melhor adaptação às condições do ambiente tropical.

Uma vez que evoluíram em ambientes quentes, os zebuínos regulam a temperatura corporal sob estresse térmico melhor que os das raças de origem Européia (Finch, 1986; Gaughan et al., 1999; Silva, 2000a). Nesses animais, os efeitos do clima tropical sobre a reprodução são menos severos que nos taurinos (Rocha et al., 1998). Em ambientes quentes, os zebuínos têm desempenho melhor que o dos taurinos sob condições nutricionais restritivas (Ferrell \& Jenkins, 1998), mas, quando comparados sob melhores condições ambientais, os zebuínos tendem a ser inferiores (Franke, 1980).

O intervalo de partos de fêmeas mais velhas foi menor que o de suas contemporâneas para o mesmo ano de nascimento. Esse resultado está relacionado ao fato de que vacas de maior idade são nascidas no início da estação e, portanto, são expostas a melhores condições de pastagem. A classe de idade entre 1.092 e 1.166 dias apresentou menor IEP, ao passo que animais com idades entre 1.750 e 1.832 dias tiveram o maior intervalo. A diferença entre as classes de idade está relacionada à maior susceptibilidade das vacas mais velhas aos problemas do aparelho reprodutor. Entretanto, Renquist et al. (2006), em estudo de cinco anos com 454 vacas cruzadas com raça britânica, observaram intervalo de partos mais longo em animais com 3 anos de idade em comparação àqueles de 4 a 9 anos de idade. Entretanto, as vacas de três anos eram primíparas e, geralmente, apresentam maior intervalo de partos. 
$\mathrm{Na}$ análise de variância do número de dias para o parto (Tabela 3), os efeitos significativos para a soma de quadrados tipo I (S.Q.I) mantiveram a mesma significância para a análise tipo III. A diferença entre estas duas análises foi o efeito significativo para o genótipo (SQIII), o que evidencia sua importância em análise após considerar todos os efeitos do modelo do número de dias para o parto. Outros efeitos significativos $(\mathrm{P}<0,05)$ foram taxa de sudação e idade da vaca.

O principal efeito sobre dias para o parto foi a idade da vaca ao parto, considerando o maior valor do quadrado médio (QM $=8.618,26$, Tabela 3$)$. Vacas com 706 a 885 dias de idade apresentaram o maior número de dias para o parto e diferiram $(\mathrm{P}<0,05)$ daquelas com 886 a 1.245 dias de idade, que tiveram a menor média, mas não diferiram significativamente entre as demais classes de idade. Essas duas classes de idade que diferiram são de fêmeas jovens e separam-se entre si por apenas 180 dias, provavelmente em virtude de diferenças no manejo ou na duração da estação de monta. Mercadante et al. (2005) também observaram diferença significativa para a idade e verificaram que o menor DPP correspondeu à maior idade da vaca (em seu estudo os animais variaram de 2 a 9 anos).

Animais com menor proporção de Hereford $(0,25 \mathrm{He}$ $0,75 \mathrm{Ne})$ tiveram menor DPP $(324,42$ dias $)$ e diferiram $(\mathrm{P}<0,05)$ das vacas 0,50 Hereford, com duas semanas a mais no DPP. Os animais $25 \%$ Hereford diferiram dos $37,5 \% \mathrm{He}$ e daqueles $62,5 \%$ Hereford. Diferença significativa entre os genótipos também ocorreu para o intervalo de partos e a maior eficiência reprodutiva foi para a maior proporção de Nelore, portanto, é vantajoso para o ambiente em estudo, em comparação aos animais com maior proporção de Hereford no mesmo ambiente.

Os zebuínos apresentam características adaptativas adequadas ao ambiente tropical em comparação aos animais de origem européia. As características do pelame e da epiderme, assim como outras, fazem parte da estrutura adaptativa dos animais ao seu ambiente. Os animais que vivem em ambientes tropicais devem possuir proteção contra a radiação solar intensa, serem capazes de eliminar através da superfície cutânea o excesso de calor corporal e, ao mesmo tempo, devem se proteger da entrada de calor externo (Silva, 2000a). A habilidade superior de termorregulação dos zebuínos em comparação aos bovinos de raças européias é resultado da baixa produção de calor metabólico e maior capacidade de perder calor para o ambiente, ou a combinação de ambos. Entretanto, as características morfológicas da epiderme dos zebuínos explicam a sua maior resistência às condições do ambiente tropical. Os aspectos adaptativos estão diretamente relacionados ao
Tabela 3 - Resumo da análise de variância de dias para o parto em vacas Braford $(n=1202)$

\begin{tabular}{lcrrl}
\hline Fonte & gl & \multicolumn{1}{c}{ SQ I } & \multicolumn{1}{c}{ SQ III } & \multicolumn{1}{c}{ QM III } \\
\hline $\mathrm{R}$ & 2 & $6.767,94^{\mathrm{NS}}$ & $3.016,98$ & $1.508,49^{\mathrm{NS}}$ \\
$\mathrm{N}$ & 2 & $3.956,44^{\mathrm{NS}}$ & $2.694,60$ & $1.347,30^{\mathrm{NS}}$ \\
$\mathrm{D}$ & 2 & $3.708,40^{\mathrm{NS}}$ & $3.884,39$ & $1.942,20^{\mathrm{NS}}$ \\
$\mathrm{C}$ & 2 & $7.606,54^{\mathrm{NS}}$ & $3.691,45$ & $1.845,72^{\mathrm{NS}}$ \\
$\mathrm{E}$ & 2 & $3.363,31^{\mathrm{NS}}$ & $5.939,28$ & $2.969,64^{\mathrm{NS}}$ \\
$\mathrm{TS}$ & 2 & $13.447,51^{* *}$ & $13.267,85$ & $6.633,92^{* *}$ \\
Genótipo & 3 & $4.914,72^{\mathrm{NS}}$ & $13.356,63$ & $4.452,21^{*}$ \\
Idade & 5 & $69.287,53^{* *}$ & $43.091,32$ & $8.618,26^{* *}$ \\
Pai & 30 & $65.982,90^{\mathrm{NS}}$ & $65.982,90$ & $2.199,43^{\mathrm{NS}}$ \\
Resíduo & 1151 & $1.855 .262,86$ & $1.855 .262,86$ & $1.611,87$ \\
$\mathrm{R}^{2}(\%)$ & 8,80 & & & \\
CV & 11,92 & & & \\
\hline
\end{tabular}

NS $P>0,05 ;{ }^{*} 0,01 \leq 0,05 ;{ }^{*} P<0,01 ; T S=$ taxa de sudação; $E=$ espessura do pelame; $\mathrm{C}=$ comprimento dos pêlos; $\mathrm{D}=$ diâmetro dos pêlos; $\mathrm{N}=$ número de pêlos por unidade de área; $R$ = refletância da superficie do pelame.

desempenho reprodutivo dos animais em ambientes específicos. De acordo com Falconer (1987), qualquer característica relacionada à fertilidade e à viabilidade do animal e de sua progênie em determinado ambiente pode estar associada à adaptação.

A taxa de sudação foi significativa $(\mathrm{P}<0,05)$ para a variação do número de dias para o parto (Tabela 3 ). Os animais com maior taxa de sudação apresentaram menor DPP, o que indica adaptação ao ambiente quente. A termólise por evaporação cutânea corresponde a $82-85 \%$ da perda total de calor nos bovinos (Gebremedhin \& Wu, 2002; Maia et al., 2005), evidenciando a importância da sudação para bovinos em ambientes quentes.

As variâncias genéticas aditivas das variáveis reprodutivas foram baixas (Tabela 4), especialmente para DPP. Os coeficientes de herdabilidade para intervalo de partos foram muito baixos de $0,05 \pm 0,04$ (no senso estrito, $\mathrm{h}^{2}$ ) e de $0,06 \pm 0,04$ (senso amplo, $\mathrm{H}^{2}$ ). A pequena diferença entre as duas estimativas indica ter sido pequeno o efeito não aditivo da composição racial, além da variância genética aditiva para o IEP, nula para o DPP. Os valores estimados foram próximos aos observados em vacas da raça Hereford, no Rio Grande do Sul, $0,01 \pm 0,06$ e $0,12 \pm 0,16$, em dois rebanhos. Valor mais alto foi observado por Silveira et al. (2004) em um rebanho de vacas Nelore no Mato Grosso do Sul.

As estimativas de herdabilidade no sentido estrito da espessura do pelame, comprimento médio dos pêlos e refletância do pelame foram menores que no sentido amplo. Os valores menores para as estimativas no sentido estrito são relacionados às diferenças não-aditivas entre os genótipos (proporção das raças Hereford: Nelore) da população em estudo, para a espessura do pelame, o comprimento médio dos pêlos e a refletância do pelame. 
A estimativa de herdabilidade do DPP foi nula. Estimativas de $\mathrm{h}^{2}$ muito baixas também têm sido relatadas para a raça Nelore no Brasil: 0,11 (Gressler et al., 2000);0,07 (Pereira et al., 2000); 0,09 (Forni \& Albulquerque et al., 2002) e 0,15 (Mercadante et al., 2005). Portanto, uma vez que IEP e DDP são características de baixa herdabilidade, apenas pequenas reduções poderão ser alcançadas por seleção massal, dependendo da intensidade da seleção aplicada.

As estimativas de herdabilidade tanto no sentido amplo como estrito $\left(\mathrm{h}^{2}=0,10\right)$ quanto no amplo $\left(\mathrm{H}^{2}=0,10\right)$ foram inferiores à relatada por Silva et al. (1988) em gado da raça Jersey $(0,22)$. As estimativas de $\mathrm{h}^{2}$ foram igualmente baixas, no entanto, as estimativas de $\mathrm{H}^{2}$ foram maiores para $\mathrm{E}(0,26 \pm 0,12), \mathrm{C}(0,39 \pm 0,15)$ e $\mathrm{R}(0,42 \pm 0,15)$. O menor valor das estimativas de herdabilidade no sentido estrito decorreram das diferenças entre os genótipos da população (proporção do Hereford), incluídas nos modelos para a estimativa da herdabilidade no sentido estrito e retiradas dos modelos para a estimativa da herdabilidade no sentido amplo. Merecem destaques as estimativas de herdabilidade para $\mathrm{R}\left(\mathrm{h}^{2}=0,30\right.$ $\left.\pm 0,12 \mathrm{eH}^{2}=0,42 \pm 0,15\right), \mathrm{C}\left(\mathrm{h}^{2}=0,18 \pm 0,09 \mathrm{eH}^{2}=0,39 \pm 0,15\right)$ e $E\left(h^{2}=0,16 \pm 0,09\right.$ e $\left.H^{2}=0,26 \pm 0,12\right)$. Maia et al. (2003) determinaram estimativas de $\mathrm{h}^{2} \mathrm{em}$ vacas da raça Holandesa maiores para $\mathrm{C}(0,38 \pm 0,14)$ e para $\mathrm{D}(0,39 \pm 0,14)$, mas valores próximos para $\mathrm{N}$ e para $\mathrm{E}$. A estimativa de $\mathrm{h}^{2}$ para $\mathrm{E}$ foi maior que a estimada por Silva et al. (1988) em vacas da raça Jersey. Na raça Holandesa, estimativas de $\mathrm{h}^{2}$ mais altas $(0,36 \pm 0,09$ e $0,37 \pm 0,07$ ) que as deste estudo foram encontradas para comprimento dos pêlos negros por Bertipaglia et al. (2007a) e Maia et al. (2003), respectivamente.

A refletância da capa do pelame poderia ser melhorada por meio da seleção, considerando a estimativa de $\mathrm{h}^{2}$ $(0,30 \pm 0,12)$; o respectivo valor da estimativa de $\mathrm{H}^{2}$ foi mais alto $(0,42 \pm 0,15)$, o que destaca a importância das diferenças entre a raça Nelore e Hereford quanto ao efeito da variância genética aditiva e não aditiva referente à composição racial incluída no modelo para a estimativa no sentido estrito e retirado para a estimativa no sentido amplo. Estas diferenças entre raças foram demonstradas por Silva et al. (2003), que determinaram a refletância de vários tipos de pelame e de epiderme em todos os comprimentos de onda entre 300 e 850 $\mathrm{nm}$ de acordo com a cor do pelame para as raças. Contudo, é importante que a seleção das características do pelame seja direcionada para obtenção de animais com pelame mais refletivo, mais denso, com pêlos curtos e grossos para favorecer as perdas de calor latente e sensível (Turnpenny et al., 2000).

Entre os resultados obtidos para as correlações genéticas entre as características da reprodução e adaptativas (Tabela 5), destaca-se a estimativa de correlação genética entre comprimento dos pêlos e o intervalo de partos, que foi alta e positiva $(0,71)$. Esse resultado sugere que a seleção para pêlos mais curtos resultará em menores intervalos de partos em animais da raça Braford. Bertipaglia et al. (2007a), no entanto, não observaram esse resultado favorável na raça Holandesa e obtiveram correlação nula entre essas duas variáveis.

De modo geral, as correlações genéticas das variáveis IEP e DPP entre as características adaptativas foram nãosignificativas $(P \geq 0,05)$. Entretanto, estas estimativas são pouco confiáveis, uma vez que seus erros-padrão foram elevados e não puderam ser expressos pelo programa MTDF. Esse problema pode ter sido originado pela herdabilidade nula de DPP ou pela falha do programa durante a estimação, em virtude da estrutura dos dados utilizados. É importante ressaltar que todos os animais tinham as medidas das características do pelame e taxa de sudação.

As estimativas de herdabilidade para a segunda variável na análise bivariada foram muito diferentes das obtidas na análise univariada. Entretanto, desde que essa análise atingiu o critério de convergência, as estimativas de herdabilidade poderiam não ser diferentes das esperadas. Espera-se que

Tabela 4 - Estimativas dos componentes de variância, herdabilidades no sentido estrito $\left(\mathrm{h}^{2}\right)$ e amplo $\left(\mathrm{H}^{2}\right)$ de análises univariadas, das características do pelame, taxa de sudação, intervalo de partos e número de dias para o parto (DPP) de vacas mestiças Nelore $\times$ Hereford

\begin{tabular}{|c|c|c|c|c|c|c|}
\hline Variável & $\sigma_{\mathrm{a}}^{2}$ & $\sigma_{\mathrm{e}}^{2}$ & $\mathrm{~h}^{2}$ & $\sigma_{a}^{2}$ & $\sigma_{\mathrm{e}}^{2}$ & $\mathrm{H}^{2}$ \\
\hline IEP & 29,85 & 530,88 & $0,05 \pm 0,04$ & 32,13 & 532,21 & $0,06 \pm 0,04$ \\
\hline DPP & 0,014 & $1.420,60$ & $0,00 \pm 0,01$ & 0,016 & $1.422,51$ & $0,00 \pm 0,01$ \\
\hline E & 0,54 & 1,54 & $0,16 \pm 0,09$ & 0,54 & 1,54 & $0,26 \pm 0,12$ \\
\hline $\mathrm{C}$ & 0,99 & 4,64 & $0,18 \pm 0,09$ & 2,39 & 3,69 & $0,39 \pm 0,15$ \\
\hline $\mathrm{N}$ & 1,86 & 21,82 & $0,08 \pm 0,06$ & 1,78 & 21,79 & $0,08 \pm 0,06$ \\
\hline $\mathrm{D}$ & 6,71 & 47,89 & $0,12 \pm 0,07$ & 6,76 & 47,82 & $0,12 \pm 0,07$ \\
\hline $\mathrm{R}$ & 0,00 & 0,01 & $0,30 \pm 0,12$ & 0,004 & 0,005 & $0,42 \pm 0,15$ \\
\hline TS & 537,85 & $5.096,88$ & $0,10 \pm 0,06$ & 552,43 & $5.083,12$ & $0,10 \pm 0,07$ \\
\hline
\end{tabular}

$\sigma^{2}{ }_{a}=$ variância genética aditiva; $\sigma^{2}{ }_{e}=$ variância ambiental; IEP = intervalo de partos; $\mathrm{DPP}=$ dias para o parto; $\mathrm{E}=$ espessura da capa de pelame; $\mathrm{C}=\mathrm{comprimento}$ dos pêlos; $\mathrm{N}=$ número de pêlos por área; $\mathrm{D}=$ diâmetro médio dos pêlos; $\mathrm{R}=$ refletância da capa de pelame; TS = taxa de sudação; As estimativas de $\mathrm{h}^{2}$ e $\mathrm{H}^{2}$ foram calculadas em análises univariadas, sendo incluídas e retiradas, respectivamente, as composições raciais dos modelos. 
Tabela 5 - Estimativas de correlações genéticas $\left(r_{g}\right)$, herdabilidade $\left(h^{2}\right)$ e erros-padrão das análises bivariadas das características do pelame, taxa de sudação, intervalo de partos (IEP) e dias para o parto (DPP) em vacas da raça Braford

\begin{tabular}{|c|c|c|c|c|}
\hline Variável 1 & Variável 2 & $r_{g}$ & $\mathrm{~h}^{2}$ da Variável 1 & $\mathrm{~h}^{2}$ da Variável 2 \\
\hline IEP & Espessura & $0,06 \pm$ & $0,05 \pm 0,04$ & $0,00 \pm 0,04$ \\
\hline IEP & Comprimento & $0,71 \pm 0,25$ & $0,07 \pm 0,05$ & $0,11 \pm 0,06$ \\
\hline IEP & Diâmetro & $0,24 \pm 0,52$ & $0,05 \pm 0,04$ & $0,08 \pm 0,04$ \\
\hline IEP & Refletância & $0,02 \pm 0,49$ & $0,22 \pm 0,10$ & $1,00 \pm 0,00$ \\
\hline IEP & Taxa de sudação & $0,00 \pm$ & $0,05 \pm 0,04$ & $0,00 \pm 0,02$ \\
\hline DPP & Espessura & $-1,00 \pm$ & $0,00 \pm 0,01$ & $0,10 \pm 0,04$ \\
\hline DPP & Comprimento & $-0,96 \pm$ & $0,00 \pm 0,01$ & $0,04 \pm 0,02$ \\
\hline DPP & Número & $1,00 \pm$ & $0,00 \pm 0,01$ & $0,02 \pm 0,02$ \\
\hline DPP & Diâmetro & $0,06 \pm$ & $0,00 \pm 0,01$ & $0,04 \pm 0,02$ \\
\hline DPP & Refletância & $-0,51 \pm$ & $0,00 \pm 0,01$ & $0,06 \pm 0,03$ \\
\hline DPP & Taxa de sudação & $0,09 \pm \ldots$ & $0,00 \pm 0,01$ & $0,00 \pm 0,01$ \\
\hline
\end{tabular}

em análises uni ou bivariadas, com medidas para todos animais e para ambas variáveis, as estimativas de herdabilidade sejam quase iguais. Os resultados deste estudo estão de acordo com outros anteriores (Silva et al., 1988; Maia et al., 2005) sobre o fato do valor elevado para a estimativa do erro-padrão.

$\mathrm{O}$ alto erro-padrão das correlações genéticas neste estudo, entretanto, não permite comparações detalhadas de todas as alternativas. Entretanto, pêlos curtos, grossos e capa de pelame pouco espessa, juntamente com perda de calor por evaporação cutânea (sudação) mais efetiva, são as melhores características para aliviar os efeitos deletérios do ambiente tropical e provavelmente resultam em melhores índices reprodutivos e produtivos.

\section{Conclusões}

As características do pelame não causam efeitos sobre o intervalo de partos e dos dias para o parto de vacas Braford. A menor capacidade de sudação está associada ao maior número de dias para o parto, o que indica inadequação desses animais ao ambiente tropical e resulta em menor desempenho reprodutivo. O intervalo de partos e o número de dias ao parto de vacas com menor proporção de Hereford foram menores, o que comprova a melhor eficiência reprodutiva e adaptação de animais com maior proporção do Nelore em ambiente tropical. Apesar do elevado erropadrão dos valores de correlações genéticas, as estimativas das correlações genéticas entre as características adaptativas do pelame e da taxa de sudação foram, de modo geral, favoráveis à seleção conjunta para melhor eficiência reprodutiva e tolerância ao calor. A seleção para pêlos mais curtos resulta em menores intervalos de partos. Novos estudos nesta área são necessários para determinar o potencial genético da tolerância ao calor na seleção para eficiência reprodutiva.

\section{Literatura Citada}

BADINGA, L.; COLLIER, R.J.; THATCHER. W.W. et al. Effects of climatic and management factors on conception rate of dairy cattle in subtropical environment. Journal of Dairy Science, v.68, n.1, p.78-85, 1985.

BARNES, F.L.; EYESTONE, W.H. Early cleavage and maternal zygotic transition in bovine embryos. Theriogenology, v.33, p.141-152, 1990

BECERRIL, C.M.; WILCOX, C.J.; LAWLOR, T.J. et al. Effects of percentage of white coat color on Holstein cow production and reproduction in a subtropical environment. Journal of Dairy Science, v.74, n.8, p.2286-2291, 1993.

BERTIPAGLIA, E.C.A.; SILVA, R.G.; MAIA A.S.C. Fertility and hair coat characteristics of Holstein cows in a tropical environment. Animal Reproduction, v.2, n.3, p.187-194, 2005.

BERTIPAGLIA, E.C.A.; SILVA, R.G.; MAIA, A.S.C. et al. Fertility and hair coat characteristics of Holstein cows in a tropical environment. Revista Brasileira de Zootecnia, v.36, n.2, p.350-359, 2007a.

BERTIPAGLIA, E.C.A.; SILVA, R.G.; CARDOSO, V. et al. Hair coat characteristics and sweating rate of Braford cows in Brazil Livestock Production Science, v.112, n.1-2, p.99-108, 2007 b.

BOLDMAN, K.G.; KRIESE, L.A.; Van VLECK, L.D. et al. A manual for use for MTDFREML. A set of programs to obtain estimates of variances and covariances. Lincoln: Department of agriculture; Agricultural Research Service, 2002. 129p.

BOURDON. R.M.; BRINKS, J.S. Calving date versus calving interval as a reproductive measure in beef cattle. Journal of Animal Science, v.57, p.1412-1417, 1983.

BUENO, R.S.; FERRAZ, J.B.S.; ELER, J.P. et al. Genetic parameters of growth and adaptive traits in a composite beef cattle population (Bos taurus x Bos indicus). In: WORLD CONGRESS ON GENETICS APPLIED TO LIVESTOCK PRODUCTION, 8., 2006, Belo Horizonte. Proceedings... Belo Horizonte: 2006. (CD-ROM).

BURROW, H.M. Variances and covariances between productive and adaptive traits and temperament in a composite breed of tropical beef cattle Livestock Production Science, v.70, p.213-233, 2001.

CENA, K.; MONTEITH, J.L. Transfer processes in animal coats. I. Radiative transfer. Proceedings of the Royal Society of London, v.188, n.2, p.377-393, 1975.

EDWARDS, J.L.; HANSEN, P.J. Differential responses of bovine oocytes and preimplantation embryos to heat shock. Molecular Reproduction and Development, v.46, p.138-145, 1997.

EDWARDS, J.A.L.; KING, W.A.; KAWARSKY, S.J. et al. Responsiveness of early embryos to environmental insults: 
potential protective roles of HSP70 and glutathione. Theriogenology, v.55, p.209-223, 2001.

FALCONER, D.S. Introdução à genética quantitativa. Viçosa, MG: Universidade Federal de Viçosa, 1987. 279p.

FERRELL, C.L.; JENKINS, T.G. Body composition and energy utilization by steers of diverse genotypes fed a high concentrate diet during the finishing period: II. Angus, Boran, Brahman, Hereford, and Tuli sires. Journal of Animal Science, v.76, p.647-657, 1998

FINCH, V.A.; BENNETT, I.L.; HOLMES, C.R. Sweating response in cattle and its relation to rectal temperature, tolerance to sun and metabolic rate. Journal of Agricultural Science, v.99, p.479-487, 1982 .

FINCH, V.A.; BENNETT, I.L.; HOLMES, C.R. Coat colour in cattle: effect on thermal balance, behaviour and growth, and relationship with coat type. Journal of Agricultural Science, v.102, p.141-147, 1984.

FINCH, V.A. Body temperature in beef cattle: its control and relevance to production in the tropics. Journal of Animal Science, v.62, p.531-542, 1986 .

FORNI, S.; DIAS, L.T.; ALBUQUERQUE, L.G. Análise genética da característica dias para o parto em bovinos da raça Nelore. Archivo Latino-Americano de Produccion Animal, v.11, p. 143-148, 2003

FRANKE, D.E. Breed and heterosis effects of American Zebu cattle. Journal of Animal Science, v.50, p.1206-1214. 1980.

GAUGHAN, J.B.; MADER, T.L.; HOLT, S.M. et al. Heat tolerance of Boran and Tuli crossbred steers Journal of Animal Science, v.77, p.2398-2405, 1999

GEBREMEDHIN, K.G.; WU, B. Simulation of sensible and laten heat losses from wet-skin surface and fur layer Journal of Thermal Biology, v.27, p.291-297, 2002.

GRESSLER, S.L.; BERGMANN, J.A.G.; PEREIRA, C.S. et al. Estudo das associações entre perímetro escrotal e características reprodutivas de fêmeas Nelore. Revista Brasileira de Zootecnia, v.29, p.427-437, 2000.

HANSEN, P.J. Embryonic mortality in cattle from the embryo's perspective. Journal of Animal Science, v.80 (E. Suppl. 2), p.E33-E44， 2002.

HANSEN, P.J. Effects of environment on bovine reproduction. In: HER, E.; WOLFENSON, D. (Eds.) Current therapy in large animal theriogenology. Philadelphia: W.B. Saunders, 1997. p.403-415.

JOHNSTON, D.J.; BUNTER, K. Days to calving in Angus cattle: genetic and environmental effects, and covariances with other traits. Livestock Production Science, v.45, p.13-22, 1996.

LEMOS, A.M.; TEODORO, R.L.; OLIVEIRA, G.P. et al. Comparative performance of six Holstein-Friesian $\times$ Guzera grades in Brazil. Animal Production, v.41, p.187-191, 1985.

LITTELL, R.C.; FREUND, R.J.; SPECTOR, P. SAS ${ }^{\circledR}$ System for linear models. 3.ed. Cary: 1991. 329p.

MAIA, A.S.C.; SILVA, R.G.; BERTIPAGLIA, E.C.A. Características do pelame de vacas Holandesas em um ambiente tropical: um estudo genético e adaptativo. Revista Brasileira de Zootecnia, v.32, n.4, p.843-853, 2003.

MAIA, A.S.C.; SILVA, R.G.; BERTIPAGLIA, E.C.A. Environmental and genetic variation of the effective radiative properties of the coat of Holstein cows under tropical conditions. Livestock Production Science, v.92, n.2, p.307-315, 2005

McARTHUR, A.J. Thermal radiation exchange, convection and the storage of latent heat in animal coats. Agricultural Forest Meteorology, v.53, p.325-336, 1991

McGREGOR, R.G. Evaluation of calving date and calving interval as measure of reproductive efficiency in beef cows. Journal of South African Veterinary Association, v.66, n.4, p.235238, 1995.

McGREGOR, R.G.; CASEY, N.H. Evaluation of calving interval and calving date as measure of reproductive performance in a beef herd. Livestock Production Science, v.57, n.2, p.181-191, 1999.
MAcMANUS, C.; PALUDO, G.R.; LOUVANDINI, H. et al. Heat tolerance in naturalized cattle in Brazil: physical factors. Archivos de Zootecnia, v.54, p.453-458, 2005

MERCADANTE, M.E.Z.; RAZOOK, A.G.; CYRILLO, J.N.S.G. et al. Parâmetros genéticos para dias ao parto, data do parto e sucesso ao parto em rebanhos experimentais da raça nelore Boletim da Indústria Animal, v.62, p.1-7, 2005.

MOREIRA, H.L.M; CARDELLINO, R.A. Herdabilidade, repetibilidade e efeitos ambientais em bovinos Hereford. II. Intervalo entre partos, habilidade materna e índice maternal produtivo. Pesquisa Agropecuária Brasileira, v.24, n.11, p.1801-1805, 1994.

NEBEL, R.L.; JOBST, S.M.; DRANSFIELD, M.B.G. et al. Use of radio frequency data communication system, HeatWatch $®$, to describe behavioral estrus in dairy cattle. Journal Dairy Science, v.80, p.179, 1997 (abstr.).

OLIVEIRA, J.F.C.; NEVES, J.P.; MORAES, J.C.F. et al. Caracterização de aspectos produtivos em vacas Brangus Ibagé com distintos graus de fertilidade. Ciência Rural, v.32, n.4, p.663-667, 2002.

ORTOVANT, R.; BOCQUIER, F.; PELLETIER, J. et al. Seasonality of reproduction in sheep and its control by photoperiod. Australian journal Biological Science, v.41, p.69-85, 1988.

PEREIRA, E.; ELER, J.P.; FERRAZ, J.B.S. Correlação genética entre perímetro escrotal e algumas características reprodutivas na raça Nelore. Revista Brasileira de Zootecnia, v.29, p.1676-1683, 2000.

PETERS, H.F.; SLEN, S.B. Hair coat characteristics of bison, domestic $\mathrm{x}$ bison hybrids, cattalo and certain domestic breeds of beef cattle. Canadian Journal of Animal Science, v.44, p.48-57, 1964.

PRAYAGA, K.C. Evaluation of beef cattle genotypes and estimation of direct and maternal genetic effects in a tropical environment. 2. Adaptive and temperament traits. Australian Journal of Agricultural Research, v.54, p.1027-1038, 2003.

PUTNEY, D.J.; MULLINS, S.; THATCHER, W.W. et al. Embryonic development in superovulated dairy cattle exposed to elevated ambient temperatures between the onset of estrus and insemination. Animal Reproduction Science, v.19, p.37$51,1989$.

RENQUIST, B.J; OLTJEN, J.W.; SAINZ, R.D. et al. Effects of age on body condition and production parameters of multiparous beef cows. Journal of Animal Science, v.84, p.1890-1895, 2006

ROCHA, A.; RANDEL, R.D.; BROUSSARD, J.R. et al. High environmental temperature and humidity decrease oocyte quality in Bos taurus but not in Bos indicus cows. Theriogenology, v.49, p.657-665, 1998.

ROCHA, J.C.M.C.; TONHATI, H.; ALENCAR, M.M. et al. Componentes de variância para o período de gestação em bovinos de corte. Arquivo Brasileiro de Medicina Veterinária e Zootecnia, v.57, n.6, p.784-791, 2005.

SARTORI, R.; SARTOR-BERGFELT, R.; MERTENS, S.A. et al. Fertilization and early embryonic development in heifers and lactating cows in summer and lactating and dry cows in winter. Journal of Dairy Science, v.85, p.2803-2812, 2002.

STATISTICAL ANALYSIS SYSTEM - SAS. User's guide: statistics version 9.0. 2.ed. Cary: SAS Institute, 2004. (CD-ROM).

SCHLEGER, A.V.; TURNER, H.G. Sweating rate of cattle in the field and their reaction to diurnal and seasonal changes. Australian Journal of Agricultural Research, v.16, p.92106, 1965.

SILVA, R.G. Improving tropical beef cattle by simultaneous selection for weight and heat tolerance: heritabilities and correlations of the traits. Journal of Animal Science, v.37, p.637-642, 1973.

SILVA, R.G. Estimativa do balanço térmico por radiação em vacas Holandesas expostas ao sol e à sombra em ambiente tropical. Revista Brasileira de Zootecnia, v.28, n.6, p.1403-1411, 1999.

SILVA, R.G. Introdução à bioclimatologia animal. São Paulo: Nobel, 2000a. 286p. 
SILVA, R.G. Um modelo para a determinação do equilíbrio térmico de bovinos em ambientes tropicais. Revista Brasileira de Zootecnia, v.29, n.4, p.1244-1252, 2000 b.

SILVA, R.G.; LA SCALA JR., N.; POCAY, P.L.B. Transmissão de radiação ultravioleta através do pelame e da epiderme de bovinos. Revista Brasileira de Zootecnia, v.30, n.6, p.1939-1947, 2001.

SILVA, R.G.; LA SCALA JR., N.; TONHATI, H. Radiative properties of the body surface of cattle and other animals. Transactions of the ASAE, v.46, n.4, p.913-918, 2003.

SILVA, R.G.; ARANTES NETO, J.G.; HOLTZ-FILHO, S.V. Genetic aspects of the variation of the sweating rate and coat characteristics of Jersey cattle. Brazilian Journal of Genetics, v.11, n.2, p.335-347, 1988.

SILVEIRA, J.C.; McMANUS, C.; MASCIOLI, A.S. et al. Fatores ambientais e parâmetros genéticos para características produtivas e reprodutivas em um rebanho nelore no estado do Mato Grosso do Sul. Revista Brasileira de Zootecnia, v.33, n.6, p.1432-1444, 2004.

TURNPENNY, J.R.; WATHES, C.M.; CLARK, J.A. et al. Thermal balance of livestock. 2. Applications of a parsimonious model. Agricultural and Forest Meteorology, v.101, p.29-52, 2000 . 